\title{
Resultados en el corto y mediano plazo del tratamiento con radiofrecuencia de la insuficiencia venosa superficial
}

\author{
Juan Bombin F. ${ }^{1,2}$, Alejandro Kotlik A. ${ }^{1,2}$, \\ Maritchu Bombin S. ${ }^{3}$ y Constanza Gómez ${ }^{1,2}$
}

'Servicio de Cirugía Hospital Dr. Eduardo Pereira. 2 Departamento de Cirugía Universidad de Valparaíso.

Unidad de Emergencia Hospital Carlos Van Buren. Valparaíso, Chile.

El autor (Dr. Juan Bombin F.) durante la ejecución del estudio perteneció al Servicio

de Cirugía del Hospital Dr. Eduardo Pereira de Valparaíso y al Departamento de Cirugía de la Universidad de Valparaíso.

Recibido el 6 de agosto de 2017 y aceptado para publicación el 23 de agosto de 2017

Correspondencia a: Dr. Juan Bombin F drbombin@gmail.com

\section{Short to midterm results after endovenous radiofrequency obliteration for treatment of saphenous reflux}

Objective: To evaluate short and midterm results after endovenous radiofrequency obliteration for treatment of saphenous reflux. Material and Method: Review and updated follow-up of a clinical series of patients after endovenous radiofrequency obliteration of insufficient saphenous trunks at the Hospital Dr. Eduardo Pereira, Valparaíso. Diagnosis was based on clinical parameters and color doppler ultrasonography. A preoperative and postoperative quality of life questionnaire was executed. Results: 77 patients (57 female) with an average age 58.3 years (23-83) and a mean follow-up time of 13.6 months. Patients consulted for symptomatic primary varicose veins of one lower extremity (49) or both (28) with 105 extremities. According to CEAP classification there were C2:84, C3:1, C4:9, C5:4 and C6:7. There were no C0 or $\mathrm{C} 1$ extremities. Superficial tributaries of more than $3 \mathrm{~mm}$ diameter were removed using Muller's method in all extremities. Furthermore, in some patients procedures were performed as complementary treatments: perforating veins ligation, skin grafting of ulcers or cutaneous-aponeurotic resection and deferred skin graft. A color doppler ultrasonography was performed one month later observing proper saphenous vein obliteration in $99 \%$ of cases and $94 \%$ at 6 -months follow-up. There were no signs of deep vein thrombosis in all patients. The quality of life questionnaire revealed symptomatic relief and surgical satisfaction in the majority of patients. Conclusion: Endovascular radiofrequency obliteration for treatment of saphenous reflux is an effective method for the short and mid terms and can be done alongside other procedure.

Key words: radiofrecuency; saphenous vein.

\section{Resumen}

Objetivo: Evaluar los resultados iniciales y en el mediano plazo del tratamiento con radiofrecuencia (RF) de la Insuficiencia Venosa Superficial (IVS) de las extremidades inferiores. Material y Método: Serie de pacientes operados en el Hospital Dr. Eduardo Pereira, Valparaíso por IVS con tratamiento de los troncos safenos insuficientes mediante ablación RF. El diagnóstico fue clínico y mediante eco-doppler color. Se efectuó un cuestionario de calidad de vida en el pre y posoperatorio. Resultados: 77 pacientes (57 mujeres), edad promedio de 58,3 años (23-83) y un seguimiento promedio de 13,6 meses. Los pacientes consultaron por várices primarias sintomáticas de una extremidad inferior (49) o ambas (28) con 105 extremidades, clasificación CEAP C2:84, C3:1, C4:9, C5:4, y C6:7. No hubo extremidades C0 o C1. Se agregó extirpación de tributarias varicosas superficiales mayores de $3 \mathrm{~mm}$ de diámetro con gancho en todas las extremidades. Además, en algunos pacientes se realizó ligadura directa de venas perforantes, injerto de úlceras, o resección cutáneo-aponeurótica e injerto diferido como tratamientos complementarios. Se realizó controles con eco-doppler color observando una correcta ablación ecográfica de la vena safena en un $99 \%$ a un mes y $94 \%$ a los 6 meses de seguimiento. Hubo ausencia de trombosis venosa profunda en todos los pacientes. El cuestionario de calidad de vida reveló en una mayoría, el alivio de los síntomas y satisfacción en relación a la cirugía. Conclusión: La ablación con radiofrecuencia de los troncos safenos insuficientes es un método efectivo en el corto y mediano plazo, que puede ser acompañado de otros procedimientos para tratar la IVS de las extremidades inferiores.

Palabras clave: ablación con radiofrecuencia; vena safena. 


\section{Introducción}

El tratamiento clásico de la insuficiencia venosa superficial, con incompetencia valvular y reflujo venoso de los troncos safenos, ha sido la crosectomía, extracción de la vena safena interna habitualmente con fleboextractor, y la flebectomía de las venas tributarias varicosas mediante múltiples incisiones. Sin embargo, comparada con otras, es una técnica relativamente traumática y se han reportado equimosis y hematomas posoperatorios en el trayecto del arrancamiento de la vena safena interna, que da lugar a dolor y dificultades de la marcha en el posoperatorio ${ }^{1}$. Además, se ha descrito hemorragias posoperatorias, lesiones del nervio safeno, linfedema, infecciones y cicatrices poco estéticas.

Existen técnicas menos invasivas que reducen estas complicaciones, entre ellas, la inyección ecoguiada de espuma esclerosante ${ }^{2,3}$, la ablación de las venas safenas con endoláser ${ }^{4} \mathrm{o}$ mediante la radiofrecuencia ${ }^{5,6}$ y últimamente obliteración de la vena safena con cianoacrilato 7 .

La ablación de los troncos safenos con el uso de radiofrecuencia consiste en la introducción de una sonda endoluminal que permite aplicar energía térmica controlada sobre la superficie interna de la pared venosa, injuria térmica que produce inicialmente una destrucción de la capa íntima y trombosis de la vena, y posterior engrosamiento y contracción de las fibras de colágeno de la capa media y adventicia, con reducción drástica del lumen e inflamación perivenosa, y finalmente, la obliteración definitiva de la vena, impidiendo de este modo el reflujo venoso patológico y la hipertensión venosa secundaria ${ }^{8,9}$.

\section{Material y Método}

Presentamos una serie clínica de 77 pacientes portadores de insuficiencia de la vena safena interna o externa tratados mediante ablación endovascular con radiofrecuencia (RF) desde el año 2014 con seguimiento actualizado al año 2016 en el Hospital Dr. Eduardo Pereira (HEP) de Valparaíso.

\section{Selección de pacientes}

Consideramos candidatos para esta cirugía a pacientes varicosos sintomáticos con clasificación $\mathrm{CEAP}^{10}$ desde $\mathrm{C} 2$ a $\mathrm{C} 6$, sin cirugía previa de várices, con diagnóstico de insuficiencia venosa crónica superficial y ausencia de insuficiencia o trombosis venosa profunda confirmada con eco-doppler color (equipo Siemmens con transductor lineal de 5 $\mathrm{MHz}$ ), ausencia de insuficiencia arterial crónica con presencia de pulsos pedios o tibiales posteriores o un Índice Tobillo/Brazo de 0,9 o mayor, movilidad adecuada de las extremidades inferiores y bajo riesgo cardiovascular. La edad, en forma aislada, no se consideró como causa de exclusión.

\section{Casuística}

Consistió en 77 pacientes con 105 extremidades tratadas (28 pacientes con ambas extremidades tratadas y 49 sólo una), 54 mujeres (70\%) y una edad promedio de 58,3 años (rango 23-83 años). Noventa y siete extremidades correspondieron a la vena safena interna con un diámetro promedio de 7,46 $\mathrm{mm}$ (rango de 4,5 a 14,5 mm), diámetro medido a $20 \mathrm{~mm}$ de la desembocadura en la vena femoral común. En ocho pacientes hubo insuficiencia de la vena safena externa con un diámetro promedio de $6 \mathrm{~mm}$ (rango de 5,5 a $6,5 \mathrm{~mm}$ ) medido a $20 \mathrm{~mm}$ de la llegada a la vena poplítea. La distribución de las extremidades según la clasificación CEAP fue de C0-C1 0, C2 84 (80\%), C3 1 (0,95\%), C4 9 (8,57\%), C5 4 (3,81\%) y C6 7 extremidades $(6,67 \%)$.

\section{Método quirúrgico}

Se realizó una revisión preoperatoria con ecodoppler color de las venas de la extremidad. Todos los pacientes fueron operados bajo anestesia regional. Siguiendo el método VNUS Closure Fast System $^{5}$ se introdujo el catéter RF dentro de la luz de la vena safena interna o externa mediante técnica de Seldinger, llevando la punta del catéter a nivel del cayado de la vena safena a unos $3 \mathrm{~cm}$ de la unión safeno-femoral o safeno-poplítea, distancia medida ecográficamente. Se infiltró abundante solución salina con inyecciones ecoguiadas hasta producir edema tumescente alrededor de la vena safena separándola de la piel, y se aplicó la energía RF por parcialidades con compresión local en posición de Trendelenburg. Se realizó control ecográfico posterior inmediato para comprobar la ablación de la vena safena y se procedió al vendaje final compresivo de la extremidad.

Además, se efectuaron procedimientos adicionales: Flebectomía de venas colaterales tributarias varicosas con un diámetro mayor de $3 \mathrm{~mm}$ con el método de Muller ${ }^{11}$ en las 105 extremidades; ligadura de venas perforantes dilatadas con diámetro mayor de $3,5 \mathrm{~mm}$ e insuficiencia valvular con reflujo mayor de 0,5 segundos en 15 extremidades; injertos inmediatos de piel en 3 pacientes con úlceras de la pierna, resección cutáneo-aponeurótica en úlceras extensas y severa lipodermatoesclerosis de la pierna con un injerto diferido de piel en 4 extremidades; y escleroterapia con polidocanol en el posoperatorio alejado en várices residuales en 30 pacientes. 


\section{Seguimiento}

Todos los pacientes operados fueron sometidos a controles posoperatorios clínicos con un promedio de 13,6 meses (Rango de 1 a 30 meses). En todos se midió la eficacia inicial del método a los 30 días y en 75 extremidades la eficacia a mediano plazo a 6 meses del posoperatorio mediante ecografía doppler color. Se observó la seguridad del procedimiento a través de la presencia o ausencia de eventos adversos graves. Se realizó también el cuestionario de calidad de vida CIVIQ2 modificado ${ }^{12,13}$ en el pre y posoperatorio a los 3 meses a 66 pacientes para me-

Tabla 1. Cuestionario de calidad de vida en tres ámbitos

1. Dolor y Molestias durante el día y al dormir

- ¿En las últimas cuatro semanas, ha sentido dolor en los tobillos y en las piernas? Indique la intensidad del dolor

- Durante las últimas cuatro semanas ¿en qué grado se sintió limitado/ molesto en su trabajo diario por el problema de sus piernas?

- Durante las últimas semanas ¿durmió mal debido al problema de sus piernas? ¿Qué tan frecuente fue?

2. Actividades cotidianas

Indique en qué grado se ha sentido molesto/limitado en las siguientes actividades:

- Mantenerse en pie largo tiempo

- Subir las escaleras

- Agacharse, arrodillarse

- Caminar enérgicamente

- Viajar en automóvil, bus, avión

- Realizar actividades domésticas, planchar, cocinar, etc.

- Hacer un deporte u otro esfuerzo físico

3. Ámbito social

- Me siento nervioso, ansioso

- Siento que soy una carga para los demás

- Me avergüenza mostrar mis piernas

- No me dan ganas de salir

- Me siento discapacitado

- Me irrito fácilmente

- Tengo dificultad para llevar a cabo las actividades matutinas

Se aplica en el pre y posoperatorio. Se responde en una escala de 1 a 5 , en que 1 es No o Nada, y 5 es Sí, Mucho o Absolutamente. dir el dolor y/o molestias durante el día y al dormir, actividades cotidianas y ámbito social y personal (Tabla 1).

\section{Resultados}

Los resultados se midieron en cuanto a eficacia, seguridad y calidad de vida.

\section{Eficacia}

La eco-doppler color inicial permitió ver la completa ablación de la vena safena interna (VSI) en 104 extremidades $(99,05 \%)$ con permeabilidad persistente en sólo 1 caso. A los 6 meses controlamos 75 extremidades, de las cuales 71 presentaron adecuada ablación de la VSI $(94,7 \%), 1$ con permeabilidad persistente $(1,3 \%)$ y 3 con permeabilidad tardía a través de una vena safena accesoria anterior (4\%). No hubo recanalización en los casos ablación de la vena safena externa.

\section{Seguridad}

Del total de 105 extremidades hubo sólo 1 caso de varicoflebitis superficial $(0,95 \%)$ y 5 casos de daño neurológico periférico $(4,76 \%)$, de los cuales 3 correspondieron a la VSI y se trató de dolor neuropático transitorio del muslo, y 2 de la vena safena externa con anestesia retromaleolar. Se encontró una importante diferencia en la aparición de neuropatía al tratar la vena safena interna o externa (Tabla 2). No hubo casos de trombosis venosa profunda ni quemaduras cutáneas ni infección del sitio operatorio.

\section{Calidad de vida}

Un cuestionario de calidad de vida se aplicó en el preoperatorio y a los 3 meses del posoperatorio en 66 pacientes. Dentro del ámbito de dolor y molestias 56 pacientes refirieron presentar una mejoría notable $(84,8 \%), 6$ escasa mejoría $(9,2 \%), 3$ sin cambios $(4,5 \%)$ y 1 empeoró $(1,5 \%)$. Respecto a las actividades cotidianas 63 pacientes refirieron mejoría

Tabla 2. Complicaciones luego de la Ablación con RF

\begin{tabular}{|lccccc|}
\hline Ablación RF & Total & Sin complicaciones & Daño neurológico & Varicoflebitis superficial & TVP \\
\hline Vena safena interna & 97 & 93 & $3(3,2 \%)$ & $1(1,0 \%)$ & 0 \\
Vena safena externa & 8 & 6 & $2(25,0 \%)$ & 0 & 0 \\
\hline Total & 105 & 99 & 5 & 1 & 0 \\
\hline
\end{tabular}

RF: Radiofrecuencia. TVP: Trombosis Venosa Profunda. No se obtuvo significación estadística entre los grupos estudiados. 
notable $(95,5 \%), 0$ escasa mejoría, 3 sin cambios $(4,5 \%)$ y ninguno empeoró. En el ámbito social y personal 61 pacientes presentaron mejoría notable $(92,4 \%), 5$ escasa mejoría $(7,6 \%)$, y nadie hubo sin cambios o empeoró.

\section{Comentario}

La enfermedad venosa de las extremidades inferiores es la enfermedad vascular más frecuente ${ }^{14}$, representando la mayor prevalencia la insuficiencia venosa crónica superficial de la vena safena interna o externa. Sin desconocer el peligro de eventos trombóticos, aparición de úlceras, eccema o episodios de varicorragia, muchas veces la enfermedad venosa es considerada un mero problema estético desconociendo los efectos en la calidad de vida de los pacientes que sufren dolores y molestias, limitaciones en las actividades cotidianas y sociales, y en su imagen corporal y autoestima personal.

El tratamiento de la insuficiencia venosa superficial debiera incluir la eliminación del reflujo valvular con la oclusión del segmento proximal de la vena safena interna o externa patológica, la extirpación de las venas colaterales varicosas dilatadas, la ligadura de las venas perforantes insuficientes y dilatadas, y la cicatrización de las zonas ulceradas de la pierna. La obliteración endovascular con radiofrecuencia de la vena safena interna o externa insuficiente y dilatada es una alternativa demostrada como ventajosa frente a la cirugía convencional con fleboextracción ${ }^{1,6,15-17}$.

Es controversial la flebectomía de venas tributarias varicosas concomitante en el mismo acto quirúrgico con la obliteración de la vena safena insuficiente o diferida ${ }^{18}$. Existen argumentos que avalan la cirugía simultánea que evita una segunda intervención ${ }^{19}$, aunque se ha publicado que una vez realizada la obliteración del tronco safeno, menos de la mitad de los pacientes vuelve a realizarse una flebectomía $^{20}$. En esta casuística preferimos efectuar estos procedimientos en una sola intervención, tomando en cuenta la idiosincrasia de nuestros pacientes, que solicitan terminar el problema en un solo tiempo quirúrgico, sin tener que volver a completar la extracción de las colaterales varicosas después, pues todos nuestros pacientes presentaron gruesas venas tributarias dilatadas sintomáticas. Siguiendo esta política realizamos también tratamiento adicional simultáneo, cuando fue necesario, ligando venas perforantes tibiales internas insuficientes y dilatadas, y también realizamos ocasionalmente cobertura a úlceras de la pierna mediante la colocación de injertos de piel en forma simultánea. Incluso, en algunos pocos casos que presentaron una severa lipodermatoesclerosis y úlcera crónica se realizó una resección cutáneo-aponeurótica y la colocación de un injerto de piel diferida con buen resultado.

Una de las complicaciones más molestas para el paciente es el daño neurológico sensitivo caracterizado por dolor urente en la cara interna del muslo y rodilla en el caso de daño neurológico al nervio safeno interno o la insensibilidad de la zona retromaleolar externa del tobillo en el caso de una injuria térmica del nervio sural. En el segundo caso el porcentaje de neuropatía fue muy alto, lo que se explica por la íntima relación del nervio sural con la vena safena externa proximal muy difícil de separar con la habitual tumefacción con solución salina. Por este motivo ya no realizamos ablación con radiofrecuencia en la vena safena externa.

Podemos desprender de nuestra experiencia que la ablación térmica mediante la aplicación de radiofrecuencia segmentaria en los troncos safenos insuficientes es efectiva, segura y tiene impacto en la calidad de vida de los pacientes operados. Puede ser además acompañado de otros procedimientos quirúrgicos simultáneos para tratar los pacientes portadores de insuficiencia venosa crónica superficial de las extremidades inferiores de una manera integral.

\section{Responsabilidades éticas}

Protección de personas y animales. Los autores declaran que los procedimientos seguidos se conformaron a las normas éticas del comité de experimentación humana responsable y de acuerdo con la Asociación Médica Mundial y la Declaración de Helsinki.

Confidencialidad de los datos. Los autores declaran que en este artículo no aparecen datos de pacientes.

Derecho a la privacidad y consentimiento informado. Los autores declaran que en este artículo no aparecen datos de pacientes.

\section{Financiación}

No existe ningún interés ni soporte financiero o comercial en este manuscrito.

\section{Conflicto de intereses}

Los autores declaran no tener ningún conflicto de intereses. 


\section{Bibliografía}

1. Lurie F, Creton D, Eklöf B, Kabnick LS, Kistner RL, Pichot O, et al. Prospective randomised study of endovenous radiofrequency obliteration (closure procedure) versus ligation and stripping in a selected patient population (EVOLVeS Study). J Vasc Surg. 2003;38:207-14.

2. Cabrera J, Cabrera J Jr, García-Olmedo A. Treatment of varicose long saphenous veins with sclerosant in microfoam form: Long-term outcomes. Phebology 2000;15:19-23.

3. González-Zeh R, Armisen R, Barahona S. Endovenous laser and echo-guided foam ablation in great saphenous vein reflux: one-year follow-up results. J Vasc Surg. 2008;4:940-6.

4. Almeida JI, Kaufman J, Gockeritz O, Chopra P, Evans MT, Hoheim DF, et al. Radiofrequency endovenous ClosureFAST versus laser ablation for treatment of great saphenous reflux: a multicenter, singleblinded, randomized study (RECOVERY study). J Vasc Interv Radiol. 2009;20:7529.

5. Rodríguez-Camarero SJ. Tratamiento endoluminal de las várices mediante radiofrecuencia VNUS-Closure ${ }^{\circledR}$ Angiología 2006;58 (Supl 2):S25-S34.

6. Merchant RF, Pichot O. Long-term outcomes of endovenous radiofrequency obliteration of saphenous reflux as a treatment for superficial venous insufficiency. J Vasc Surg. 2005;42:502-9.

7. Almeida JI, Javier JJ, MacKay EG, Bautista C, Cher DJ, Proebstle TM.
Two-year follow-up of first human use of cyanoacrylate adhesive for treatment of saphenous vein incompetence. Phlebology 2015;30:397-404

8. García-Madrid C, Pastor JO, Gómez F y Sala E. Nuevos avances en el tratamiento de las várices: radiofrecuencia endovenosa VNUS Closure. Cir Esp. 2011;89:420-26.

9. Schmedt CG, Sroka R, Steckmeier S, Meissner OA, Babaryka G, Hunger K, et al. Investigation on radiofrequency and laser $(980 \mathrm{~nm})$ effects after endoluminal treatment of saphenous vein insufficiency in an exvivo model. Eur J Endovasc Surg. 2006;32:318-25.

10. Eklöf B, Rutherford RB, Bergan JJ, Carpentier IH, Gloviczki P, Kistner LL et al. Revision of the CEAP Classification for chronic venous disorders: Consensus statement. J Vasc Surg. 2004;40:1248-52.

11. Muller R. Traitement des varices par la phlébectomie en ambulatoire. Phlébologie 1966;19:277-9.

12. Launois R, Reboul-Marty J, Henry B. Construction and validation of a quality of life questionnaire in chronic lower limb venous insufficiency (CIVIQ). Qual Life Res. 1996;5:539-54

13. Guexx JJ. L'evaluation des résultats dans le traitment des varices. Phlébologie 2008; 37:205-9.

14. Adhikari A, Criqui MH, Wooll V et al. The epidemiology of chronic venous diseases. Phlebology 2000;15:2-18.

15. Perala J, Rautio T, Biancari F, Ohtonen P, Wiik, Heikkinen T, et al. Radiofrequency endovenous obliteration versus stripping of the long saphenous vein in the management of primary varicose veins:
3 -year outcome of a randomized study. Ann Vasc Surg. 2005;19:669-72.

16. Rasmussen L, Lawaetz M, Serup J, Bjoern L, Vennits B, Blemings A, et al. Randomized clinical trial comparing endovenous laser ablation, radiofrecuency ablation, foam sclerotherapy, and surgical stripping for great saphenous varicose veins with 3-year follow-up. J Vasc Surg. 2013;1:349-56.

17. Gloviczki P, Comerota AJ, Dalsing MC, Eklof BG, Gillepie DL, Gloviczki ML, et al. The care of patients with varicose veins and associated chronic venous diseases: clinical practice guidelines of the Society for Vascular and the American Venous Forum. J Vasc Surg. 2011;5 (5 Suppl):2S$48 \mathrm{~S}$.

18. Proebstle TM, Alm J, Göckeritz O, Wenzel C, Noppeney T, Lebard C, et al. Three-year European follow-up of endovenous radiofrequency-powered segmental thermal ablation of the great saphenous vein with or without treatment of calf varicosities. J Vasc Surg. 2011;54:146-52.

19. Harlander-Locke M, Jiménez JC, Lawrence PF, Derubertis BG, Rigberg DA, Gelabert HA. Endovenous ablation with concomitant phlebectomy is a safe and effective method of treatment for syntomatic patients with axial reflux and incompetent tributaries. J Vasc Surg. 2013;58: 166-72.

20. Schanzer H. Endovenous Ablation plus Microphlebectomy/Sclerotherapy for treatment of varicose veins: single or two stage procedure. Vasc and Endovasc Surg. 2010;44:545-9. 